\title{
Recognition of sports action pattern based on sparse matrix vector Song Yupeng ${ }^{1}$, Jia Ling ${ }^{1}$ \\ 1.Zhuhai College of Jilin University, Guangdong Zhuhai, 519000, China
}

Keywords: recognition of sport action; sparse matrix vector; optical-flow features; pattern recognition

\begin{abstract}
The Thesis proposes an algorithm of physical gesture recognition of human body based on composite features, which combines the feature of contour vector distance from the contour mass center to the edge point with the optical-flow features, and then the composite features are formed to make action recognition. According to the recognition results in the third part, we can see that such composite features achieve more than 95\% correct recognition rate in the Weizmann database and KTH database, which proves the validity and feasibility of such algorithm sufficiently.
\end{abstract}

\section{Introduction}

The sports action information in the video sequence generally can be described in features of optical flow, silhouette and interest points etc. Based on the sufficient consideration of advantages and disadvantages as well as applicable scope of different features, new composite features combining the static features and local optical-flow features described in global shape have been proposed. Firstly, the approximate location of sports action is decided with background subtraction, then the human body silhouette is obtained, and the overall information of the human body appearance can be expressed with vector of silhouette contour. Then in the sport area, the optical flow shall be extracted, and the local optical-flow information is used to indicate the local features of the human body actions, so as to improve the anti-noise capacity of the optical flow. At last, the combination of the overall silhouette features and local optical-flow features shall be taken as the composite features. The experiment indicates that the robustness and recognition performance of composite features are better than the single feature.

\section{Feature expression and extraction}

\section{Image preprocessing}

The image preprocessing can reduce the image area and processing data of the research and reduce the calculated amount. Generally, it uses the background subtraction to decide the approximate area and body silhouette of sports action, as shown in Fig. 1(b). It assumes that all sports actions are carried out in front of the statistic background, according to the silhouette information, and the confirmable interest region is shown in Fig. 1 (c). After the recognition of region of interest, then we can only dispose the information within the region of interest.

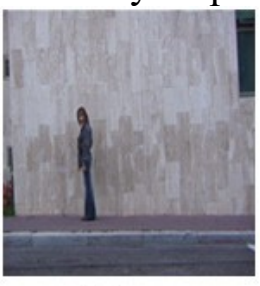

(a)

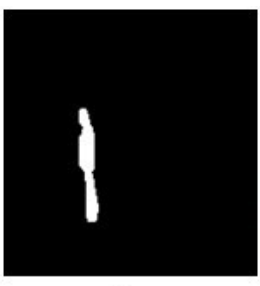

(b)

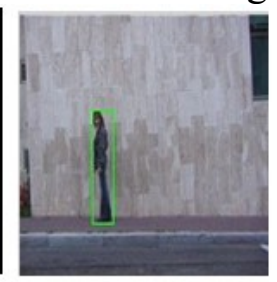

(c)

Fig 1 (a) Image of Original Frame in Video; (b) Body Silhouette; (c) Image of Original Frame Marked

Expression and extraction of silhouette features

In the image of single frame, the body silhouette can be used to describe the information of entire shape changes of human body actions. The selection of silhouette features has the following features: (1) the silhouette features can describe the shape information of moving body easily and 
intuitively; (2) the silhouette features can be extracted easily; (3) the binary silhouette figure has no sensitivity to the texture and color of the foreground image. This step aims on transferring the sports action information of the original video into the relevant morphological characteristics sequences, and these sequences indicate the process of moving process.

Given: a sports video $V$ has image $I$ of $T$ frame, as shown in equation $V=\left[I_{1}, I_{2}, \cdots, I_{T}\right]$, the corresponding sports-action silhouette sequences shall be $S=\left[\mathrm{s}_{1}, s_{2}, \cdots, s_{T}\right], S$ has been obtained in the preprocessing of the image. For the sake of simplicity, we use the method of contour vector [7] to describe the overall shape information of the body silhouette. The specific process shall be as follows:

(I) Use the canny edge detector to obtain the edge contour of silhouette of each frame, which is shown in Fig. 2 (a), and the coordinates of such edge contour shall be as shown in Fig. 2 (b). Therefore, the body contour can be expressed in $n_{t}$ point, which shall be $\left\{\left(x_{1}, y_{1}\right),\left(x_{2}, y_{2}\right), \cdots,\left(x_{n_{t}}, y_{n_{t}}\right)\right\}, t=1,2,3, \cdots T$.

(II) The mass center of the human body shall be obtained with equation (1).

$$
\left(x_{c}, y_{c}\right)=\left(\frac{1}{n_{t}} \sum_{i=1}^{n_{t}} x_{i}, \frac{1}{n_{t}} \sum_{i=1}^{n_{t}} y_{i}\right)
$$

Where, $\left(x_{c}, y_{c}\right)$ is the mass center, $\left(x_{i}, y_{i}\right)$ is the edge point of the contour, and $n_{t}$ is the number of edge points of No. $t$ image.

(III) The distance from the mass center to the edge point can be obtained with equation (2).

$$
d_{i}=\sqrt{\left(x_{i}-x_{c}\right)^{2}+\left(y_{i}-y_{c}\right)^{2}}, i=1,2, \cdots, n_{t}
$$

$d_{i}$ is the distance form the mass center corresponding to No. $i$ edge point to the edge point. In calculation, it starts from the most point on the left and the top of the contour figure, and calculate in order in clockwise, then the contour vector $D_{t}$ of one dimension can be obtained from the two-dimensional contour figure of single-frame image $I_{t}$.

$$
D_{t}=\left[d_{1}, d_{2}, d_{3}, \cdots, d_{i}, \cdots, d_{n_{t}}\right], i=1,2, \cdots, n_{t}
$$

(IV) In order to eliminate the influence of spatial scale and distance, use 2-norm to implement normalization processing on the vector. As the sizes of edge points $n_{t}$ of images in each frame are different, the contour vector shall receive resample in equal interval after normalization processing to get the number $\mathrm{N}$ of fixed points. The Thesis shall implement experiments on different valve $\mathrm{N}$, which indicates that the calculated amount of $\mathrm{N}=200$ is less, and it can also indicate sports action information completely. Both single feature and composite features can achieve the highest recognition rate. When the sampling point is $\mathrm{N}=200$, the contour vector results shall be as shown in Fig. 2 (c). 


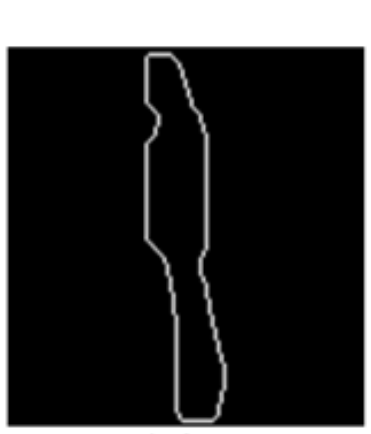

(a)
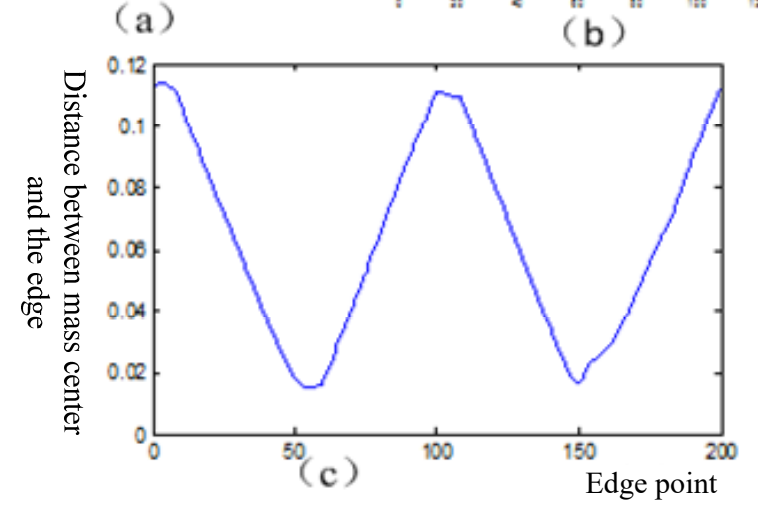

Fig. 2 (a): Silhouette Contour Figure; (b) Silhouette Contour Figure Brought into Coordinate; (c)

\section{Contour Vector Features Obtained finally}

Expression and extraction of optical-flow features

The inaccuracy of silhouette image may cause the contour vector feature information not to express the action features accurately. At this time, the optical-flow features can express the action information in the video sequence effectively and accurately. Extract the optical flow within the sports action region, and use the local optical-flow information of the region to express the local features of human body sports action, thus the anti-noise capacity of the optical flow can be improved. The specific extraction and expression process of optical flow shall be as follows:

(I) Decide the region of interest corresponding to image $I_{t}$ of current frame, cut the current frame corresponding to such region of interest and gray-scale image area corresponding to the previous frame, as shown in Fig. 3 (a1) and (a2). Then the method of Lucas - Kanade shall be used for optical-flow detection in the region of interest of current frame and the previous frame, from which the optical flow filed gotten shall be as shown in Fig. 3 (b), and the optical flow shall be divided into two vectors of vertical and horizontal, which shall be vertical optical flow and horizontal optical flow.

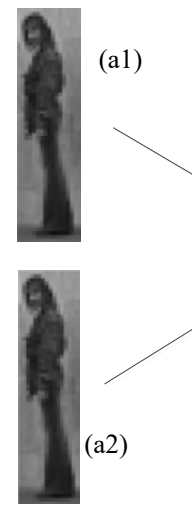

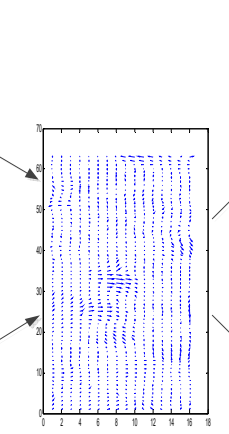

(b)

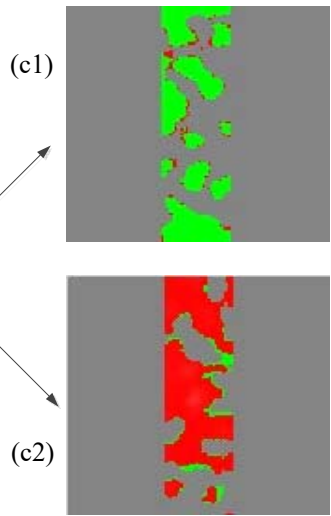

Fig. 3 (a1) Grey Scale Image of Interest Region of t-1 Moment; (a2) Grey Scale Image of Interest Region of $\mathrm{t}$ Moment; (b) Optical Flow Filed of t Moment; the optical flow field is divided into two directions; (c1): Optical Flow Image in Vertical; (c2) Optical Flow Image in Horizontal.

(II) To reduce the information dimensions of optical flow information and find out the data 
expression with identification ability, the method of sub-regional radial histogram is used to make statistics of optical flow features. Firstly, with the premises of long side scaling, the optical flow image in the region of interest shall be standardized into the optical flow image with unified size in $120 * 120$ dimensions, as shown in Fig. 3 (c1) and (c2). The standardized optical-flow image shall be divided into sub-frame $S_{1}, S_{2}, S_{3}, S_{4}$ of $2 * 2$, and the sub-frame shall be $60 * 60$, where, the center points shall be $C_{1}, C_{2}, C_{3}, C_{4}$ separately, as shown in Fig. 4 (a). Then the center point of the sub-frame shall be center for dividing the sub-frame into 18 sub-regions, which are $^{S_{i, 1}}, S_{i, 2}, \cdots, S_{i, 18}, i=1,2,3,4$, as shown in Fig. 4 (b), each central angle shall take 200, which shall form 72 sub-regions.

(III) In the sub-region ${ }^{S_{i j}}$, there are $k$ optical flows in vertical (or optical flows in horizontal), add all optical flows in vertical (or optical flows in horizontal) shall get the sum $O_{L i, j}$ of optical flow in vertical (the sum $O_{H i, j}$ of optical flows in horizontal) in region $S_{i j}$. The equation (4) and (5) shall calculate the sum of optical flow in vertical and the sum of optical flows in horizontal of the sub-region separately.

$$
\begin{aligned}
& O_{L i, j}=\sum_{m=1}^{k} O_{L m},\left(x_{O_{L m}}, y_{O_{L m}}\right) \in S_{i, j} \\
& O_{H i, j}=\sum_{m=1}^{k} O_{H m},\left(x_{O_{H m}}, y_{O_{H m}}\right) \in S_{i, j}
\end{aligned}
$$

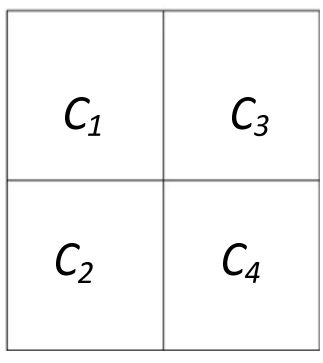

(a)

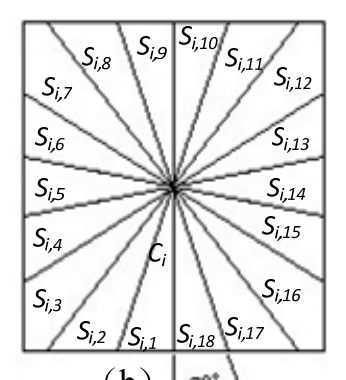

(b) $3 e$ -

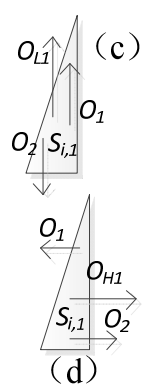

(d)

Fig. 4 (a): 2*2 Sub-frame Image in Sports Region; (b) Sub-frame Region Divided into 18 Sub-regions, the centric angle of each sub-region shall be 200; (c) Optical Flow in Vertical in Sub-region; (d) Optical Flow in Horizontal in Sub-region

\section{Experiment analyses}

Database introduction

This experiment is achieved within the operation in MATLAB2010a. There are ten actions in the Weizmann action video database, which are Bend, Jack, Jump, Pjump, Run, Side, Skip, Walk, Wave1 and Wave2, each action is completed by 9 people. The video background and angle of view have no changes, and the resolution ratio of image in each frame is $144 * 180$, the frame rate is 25 frame/s, the example is as shown in Fig. 5. There are 6 actions in KTH database, which are Boxing, Handclapping, Handwaving, Jogging, Running and Walking, each action is completed by 25 different people in four scenarios, there are 599 videos in total. The background is in relative rest, except the zoom in and out of the shot, the sports action of the camera is relatively slight, as shown in Fig. 5. 

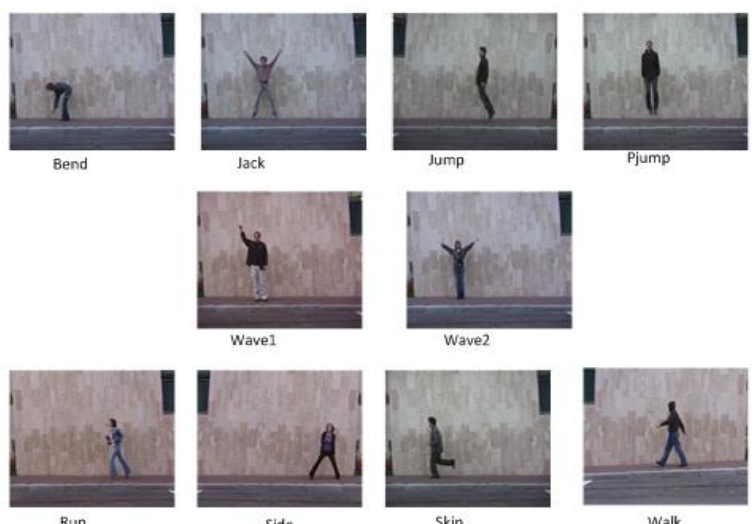

Fig. 5 Examples of Ten Actions in Weizmann Database

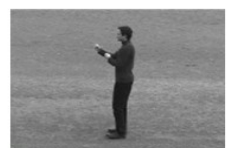

Boxing

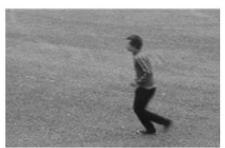

Jogging

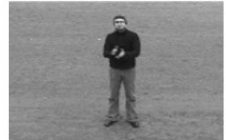

Handclapping

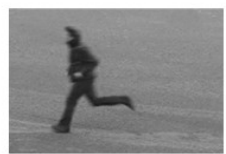

Running

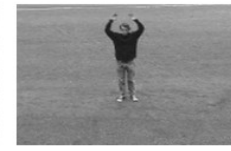

Handwaving

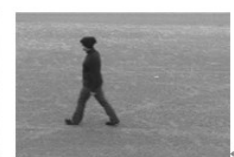

Walking

Experiment and result analysis

Fig. 5 Examples of Six Actions in KTH Database

This Thesis adopts the methods in the first part to extract the contour vector features, optical-flow features and composite features of human body sports action to represent the actions. To achieve the accuracy of unbiased estimation, the method of leaving one out is adopted to verify the experiment results, which means that all sports actions completed by one person in the database selected in each experiment shall be taken as the test sample, and the rest shall be the training sample set. Then the circulation shall be carried out, the actions of each person shall be taken as the test sample for test, and the statistics of recognition results shall be implemented. The method of nearest neighbor is adopted for classified recognition. The recognition results of optical-flow features, contour distance features and composite features of their combination are as shown in the following Table 1.

Table 1 Select the Recognition Rate Corresponding to Different Features

\begin{tabular}{|cccc|}
\hline $\begin{array}{c}\text { Features } \\
\text { Recognition rate } \\
\text { Weizmann }\end{array}$ & $\begin{array}{c}\text { Contour vector } \\
\text { features }\end{array}$ & $\begin{array}{c}\text { Optical-flow } \\
\text { features }\end{array}$ & $\begin{array}{l}\text { Composite } \\
\text { features }\end{array}$ \\
$\begin{array}{c}\text { Weizmann } \\
\text { database } \\
\text { KTH database }\end{array}$ & $88.89 \%$ & $98.89 \%$ & $100 \%$ \\
\hline
\end{tabular}

According to Table 1, the recognition rate of composite features is higher than that of single feature, and $100 \%$ right recognition rate can be obtained from Weizmann database; the recognition rate in KTH database can reach $95 \%$.

\section{Conclusion}

The selection of sports action features can affect the recognition effect of body action recognition methods directly. The single feature is under different influences of human body appearance, environment, and camera setup etc, and its applicable scope is also different, and the recognition effect is limited. On the basis of studying the presentations and recognition of human body actions, the advantages and disadvantages of different features have been taken into sufficient consideration, and the composite features in the combination of overall silhouette features and local optical-flow features have been proposed, and used in the recognition of human body actions. The experiment results indicate that such algorithm has achieved the ideal results, and $100 \%$ of right recognition rate on the actions in Weizmann database can be achieved. 


\section{Reference}

[1] Yan L. Models of regional agro-climatic resources survey and system assessment based on geo-spatial data mining.[C]// IEEE International Conference on Spatial Data Mining and Geographical Knowledge Services, Icsdm 2011, Fuzhou, China, June 29 - July. 2011:435-439.

[2] Meyssignac B, Becker M, Llovel W, et al. An Assessment of Two-Dimensional Past Sea Level Reconstructions Over 1950-2009 Based on Tide-Gauge Data and Different Input Sea Level Grids[J]. Surveys in Geophysics, 2012, 33(5):945-972.

[3] Wang T, Guan L. A data mining technique based on pattern discovery and k-nearest neighbor classifier for transient stability assessment[C]// Power Engineering Conference, 2007. IPEC 2007. International. 2008:118-123.

[4] Yan W. Application Research of Data Mining Technology about Teaching Quality Assessment in Colleges and Universities[J]. Procedia Engineering, 2011, 15:4241-4245.

[5] Swart P, Dewulf J. Corrigendum to "Quantifying the impacts of primary metal resource use in life cycle assessment based on recent mining data" [Resour. Conserv. Recycl. 73 (2013) 180-187][J]. Resources Conservation \& Recycling, 2014, 82:31-47. 